\title{
Infant and young child feeding counseling: an intervention study
}

\author{
Katia Cristina Bassichetto, ${ }^{1}$ Marina Ferreira Réa
}

\begin{abstract}
Objective: To evaluate the effectiveness of an integrated infant and young child feeding counseling course for transforming the knowledge, attitudes and practices of pediatricians and nutritionists working for the municipal health system of São Paulo, Brazil.

Methods: A randomized intervention study enrolling 29 professionals in the intervention group and 27 in the control group. Interviewers were trained in advance to collect data on the professionals working at health centers, before and 2 months after the intervention. Three research instruments were used, the first was to assess the profile of each professional, the second assessed their knowledge and the third was a clinical observation protocol. Analysis was performed using the Kruskal-Wallis test for independent samples and the Tukey method.
\end{abstract}

Results: The results for the knowledge questionnaire showed improvements in the intervention group $(p<0.001)$ for the whole questionnaire and for questions on breastfeeding ( $p=0.004)$; HIV and infant and young child feeding ( $p$ $=0.049)$; complementary feeding $(p=0.012)$; and counseling in infant and young child feeding $(p=0.004)$. In terms of performance, it was observed that the intervention group had significantly improved their dietary anamnesis after the intervention $(p<0.001)$.

Conclusions: This course effectively promoted an increase in knowledge and improvements in dietary anamnesis performance, but the same was not true of counseling skills.

J Pediatr (Rio J). 2008;84(1):75-82: Child nutrition/education, health personnel, intervention studies, effectiveness, breastfeeding.

\section{Introduction}

Several initiatives have been implemented to retrain health professionals in counseling, which is a technique that can be described as a process of active listening, that is personalized and client-centered, and which presupposes the capacity to establish a relationship of trust between the interlocutors, aiming to recover internal resources in order that they are able to see themselves as subject to their own health and transformation. Stimulation of the individual's autonomy so that they can solve their problems is related, to a certain extent, the Theory of Client-Centered Counseling, developed by Carl Rogers. ${ }^{1}$ It includes three components: emotional support, educational support and risk assessment, favoring reflection on values, attitudes and behavior related to the subject being dealt with. ${ }^{2}$ This methodology, as applied to training on infant and young child feeding, aims to strengthen mothers or carers so that they become capable of taking the correct decisions with respect to children, and is the basis of

1. Doutora. Secretaria Municipal da Saúde de São Paulo, São Paulo, SP, Brazil.

2. Doutora, Departamento de Medicina Preventiva, Universidade de São Paulo (USP), São Paulo, SP, Brazil. Instituto de Saúde, Secretaria de Estado da Saúde de São Paulo, São Paulo, SP, Brazil.

Financial support: This project received grants from Fundação de Amparo à Pesquisa do Estado de São Paulo (FAPESP), protocol no. 02/07836-2, and from the World Health Organization (WHO), protocol no. HQ/01/108/103.

The present study is part of the doctoral thesis entitled "Aconselhamento em alimentação infantil - avaliação de uma proposta da Organização Mundial da Saúde para capacitação de profissionais de saúde da cidade de São Paulo" (Infant and young child feeding counseling: evaluation of a Wold Health Organization proposal for the training of health professionals in the city of São Paulo), presented at the Graduate Program in Sciences, Coordenadoria de Controle de Doenças, Secretaria de Estado da Saúde de São Paulo, São Paulo, SP, Brazil, in August 2006.

No conflicts of interest declared concerning the publication of this article.

Suggested citation: Bassichetto KC, Réa MF. Infant and young child feeding counseling: an intervention study. J Pediatr (Rio J). 2008 ;84(1):75-82.

Manuscript received Sep 21 2007, accepted for publication Nov 212007.

doi:10.2223/JPED. 1750 
three World Health Organization/ United Nations Children's Fund (WHO/UNICEF) courses: breastfeeding counseling, ${ }^{3} \mathrm{HIV}$ and infant feeding counseling ${ }^{4}$ and complementary feeding counseling. 5

These are being implemented in several countries, after having been tested in different cultures (South Africa, Ghana and Jamaica), with the objective of making them appropriate for widespread use. To further facilitate their use, the WHO recently took the initiative of collecting their content into a single course entitled, "Infant and young child feeding counseling: an Integrated Course." and which will be referred to here as the, "Integrated Course." ${ }^{6}$ Several different studies $^{7-16}$ have analyzed courses that included the subject of counseling, with favorable findings, but all of them were dealing with breastfeeding only.

The Integrated Course brings together information on the complementary feeding of children from 6 to 24 months, on feeding children of HIV-positive mothers and on breastfeeding.

The course also contains other elements of the theory of human communication and professional-client interpersonal relationships, as instrumental in providing guidance on healthy infant and young child feeding practices, substituting models in which professionals are the repositories of knowledge and end up establishing relationships in which they have power over the patient, impeding effective therapeutic communication. ${ }^{17}$ There are many references to investment in educational processes, focusing on improving professionalclient communication as a basis for the development of, "the skills for good listening and learning." ${ }^{18-21}$

In practice, health professionals have certain difficulties in keeping up-to-date with relation to infant and young child feeding, especially due to the lack of investment by the public sector in training that focuses on improving counseling performance. In our view, in the case of the public health system, the constant pressure to meet demands for care has made management unreceptive to experimentation in the field of permanent education, and even more so if they are unable to anticipate clear, practical results.

It was this context that prompted the study described here, the objective of which is to evaluate the effectiveness of the Integrated Course for transforming the knowledge, attitudes and practices of health professionals specialized in sexually transmitted diseases (STDs)/AIDS or working for the primary care services of the municipal health department of the city of São Paulo. This article attempts to analyze whether the training provided by the Integrated Course allows counselors to bring themselves up-to-date on infant and young child feeding and, at the same time, prepare themselves adequately to utilize counseling skills, without interfering in the life of each individual and supporting them in finding solutions that are realistic in their situation.

\section{Methods}

An intervention study was carried out in 2005 with a sample of health professionals who took the Integrated Course - intervention group (IG) - and a sample who did not - control group (CG) - all employed by the public health system of the city of São Paulo, Brazil. These professionals were assessed at two points: before, and 2 months after, intervention, with one consultation from each professional observed prior to intervention and two consultations observed post intervention.

Since the methodology of the Integrated Course limits the number of participants in each course to 24 to 30 , our sample could not be larger than 30 . In order to take account of possible losses, it was decided that the sample size should be around 40 cases (IG) and 40 controls (CG). This maximum was, therefore, set by the methodology of the course (which aims to make sure that everyone benefits from practical lessons).

The inclusion criteria in selecting the professionals were: pediatricians or nutritionists involved in caring for children up to 24 months of age, with permission from their management to take part and, in the case of the pediatricians, where another professional was available during the same shift. These professionals were selected from those working at services provided by the Department of Health (Secretaria de Saúde) of the city of São Paulo (DH/SP), providing primary care or specializing in STD/AIDS and where the Family Health Strategy has not been implemented, due to the fact that the professionals working at services where this has been implemented have already received increased investment in training in general.

All of the eligible health centers in the city were contacted, a total of 147: 132 Basic Health Units (UBS) and 15 STD/AIDS clinics, 63 of which agreed to participate (49 UBS and 14 specialized clinics). This represents $43 \%$ of the total, and, from these clinics, 74 professionals received permission to participate. These were then allocated by lots into the study groups, 37 in each. A variety of reasons were given for not participating in the project: the distance from the place where the course was to be held, holidays, illness and others. After being informed of the results of the randomization, some participants dropped out, leaving 31 in IG and 28 in CG (losses were due to change of jobs, health problems, permission being revoked or because they had not been chosen to take the course).

After the course, at the second observation point, there were further losses: two from IG and one from CG, due to refusal or because the participants could not be located after several attempts. In all, there were 18 dropouts (24.3\%), eight from IG (21.6\%) and 10 from CG (27.0\%), three from specialized clinics and 15 from UBS, making a total of 15 pediatricians ( $83 \%$ ) and three nutritionists (17.3\%). In order to verify the validity of the randomization process, certain 
characteristics of these professionals were compared between groups, in order to check if there were similarities between CG and IG, by applying statistical tests to some of the preintervention study variables: sex, age, time since qualification and length of experience with infant and young child feeding. Fisher's exact test was used for discrete variables and the Kruskal Wallis test for continual variables.

In order to apply the intervention, the teaching materials for the Integrated Course (guides for the director, facilitator and participants) were translated and adapted for the Brazilian context. The course includes 8 hours of practical sessions ( 4 in a maternity unit, 2 in a kitchen facility and 2 at a clinic). There are a total of 34 sessions, 8 of which are dedicated to breastfeeding, 6 to HIV and infant and young child feeding, 7 to complementary feeding 10 to counseling and 3 to general themes on infant and young child feeding, making a total of 40 hours of training.

The training team was made up of one director (a pediatrician with expertise in carrying out these courses) and 11 facilitators (pediatricians, nutritionist, nurses and psychologist) who met the pre-requisites of having taken counseling courses.

Three data collection instruments were used, all applied at the health services: the first was a questionnaire on the profile of each professional (PPQ) - self administered, with both open and closed questions on training, professional experience and working conditions; the second was a questionnaire on the practice of counseling on infant and young child feeding (IFCQ) - multiple choice questions; the last instrument was a protocol for observation of clinical consultations (CCOP) - recording the presence and frequency of certain actions during the consultation.

Interviewers had previously taken a seven-hour training course including both theory and practice, including testing and application of the instruments and, on a later date, practice consultation observations by pairs of interviewers, each blind to the scoring of the other, aimed to measure the degree of variability or concordance between them. At a later meeting, interviewers were chosen based on consistency of evaluation and how they filled out the CCOP.

For some actions or groups of actions, scores were formulated by which the value of 1 was awarded to participants who carried out all actions of a particular group and 0 to participants who failed to perform at least one of them. These were: anthropometry - weighing, measuring and filling in graphs; dietary anamnesis - frequency, quantity, consistency, preparation, who feeds and how they feed, guidance and demonstration on feeding; performance - the sum of all scores attributed in the questionnaire to all the items in the section, "Personal characteristics of the professional during the consultation," where the lower the score, the better the performance, with possible scores from 0 to 82 ; feeding - the sum of all scores attributed to all the items in the section, "Questions on feeding," variation: 0 to 22; actions - the sum of all scores attributed to all the items in the section, "Actions," variation: 0 to 18; behavior of mother - the sum of scores attributed to the section, "Personal characteristics of the mother or carer during the consultation," variation: 0 to 15 .

In order to maintain quality control, interviewers were kept blind to the results of randomization, although some professionals ended up revealing their status - intervention or control; the interviewers were selected carefully and "calibrated" for consistency of observation of a single consultation, pre-tested and standardized instruments were used and data were double-input to the database.

In order to analyze the professionals' knowledge, scores were attributed to each of the four topics in the IFCQ breastfeeding; HIV and infant and young child feeding; complementary feeding and counseling, plus an overall score. These scores were compared by time of assessment (1 immediately prior to the course and 2 - after the course, immediately after and 2 months after) and by group (IG or $\mathrm{CG}$ ). For each participant the differences between the prior scores and the mean of the two post-intervention scores were calculated: if the difference was less than zero, this signified an increase in knowledge, if greater than zero, this indicated a loss of knowledge and, if zero, there had been no change. These scores were described, by group, as minimum and maximum values and the median of the differences. The Kruskal-Wallis test was used for independent samples (CG vs. IG). Results where $p<0.05$ were considered statistically significant.

For the analysis of performance, according to the CCOP scores, the items observed were classified into four domains: A - counseling skills; B - dietary anamnesis; C - nutritional assessment and guidance; and $D$ - reaction of mother to professional. A score was attributed to each domain to represent a synthesis of what was expected for each theme. For example, for the counseling skills domain, scores varied from 1 (constantly) to 5 (never) and the mean score of observations was taken for each domain.

In order to compare the behavior of the groups, we employed analysis of variance for repeated measures, by observation point ( 1 or $2^{*}$ ) and by group (CG or IG). When necessary, the Tukey method was used for multiple comparisons. ${ }^{22}$ Considering the weightings given to each possibility of classification of the observation - 1 (constantly) to 5 (never); 0 not assessed -, the lower the score, the better the professional's assessment.

This project was approved by the Research Ethics Committee of the DH/SP. Free and informed consent forms were signed by the professionals and the mothers. 


\section{Results}

The results below relate to 56 professionals: 29 in IG and 27 in CG. The variations in some of the totals are the result of incomplete information.

When verifying the randomization, no differences were identified between IG and CG for any of the variables analyzed (Table 1). Therefore, it is considered that, despite the losses, the process was adequate and resulted in two similar groups.

Of the 56 participants who remained at the end of the study, 23 are nutritionists and 33 are pediatricians. The majority $(94.6 \%)$ are female, and the predominant age group was from 40 to 49 years (66\%). A significant proportion of these participants $(64.3 \%)$ have been trained for more than 21 years, having a great deal of experience with infant and young child feeding, and $64.3 \%$ have worked in the area for more than 11 years. The proportion of individuals whose knowledge improved was greater in the IG $(89.7 \%)$ than in the CG $(33.3 \%)(p<0.001)$.

It was observed that the proportion of individuals whose knowledge had increased occurred in all topics assessed: breastfeeding - IG (79.3\%) and CG (37\%) ( $p=0.004)$; HIV and infant and young child feeding - IG (48.3\%) and CG $(18.5 \%)(p=0.049)$; complementary feeding - IG (69.0\%) and CG $(37.0 \%)(p=0.012)$; counseling - IG (51.7\%) and CG $(22.2 \%)(p=0.004)$, as illustrated by Figure 1 , and statistically significant difference were observed between the groups. Multiple comparisons indicated statistically significant difference between the groups at the second point (postintervention) and between observation points as well, 1 and $2 *$, in the IG. In all of these cases $p<0.001$ was observed.

In order to compare the performance of the professionals during clinical consultation, graphs of mean profiles were build.

Table 1 - Comparison between control and intervention groups for some of the discrete and continual variables (São Paulo, SP, Brazil, 2005)

\begin{tabular}{|c|c|c|c|c|c|c|c|}
\hline \multirow[b]{3}{*}{ Discrete variables } & \multicolumn{4}{|c|}{ Groups } & & & \multirow[b]{3}{*}{ p* } \\
\hline & \multicolumn{2}{|c|}{ Control } & \multicolumn{2}{|c|}{ Intervention } & \multicolumn{2}{|c|}{ Total } & \\
\hline & $\mathbf{n}$ & $(\%)$ & $\mathbf{n}$ & $(\%)$ & $\mathbf{n}$ & $(\%)$ & \\
\hline \multicolumn{8}{|l|}{ Sex of professional } \\
\hline $\mathrm{F}$ & 26 & $(92.9)$ & 28 & $(90.3)$ & 54 & $(91.5)$ & \\
\hline M & 2 & $(7.1)$ & 3 & $(9.7)$ & 5 & $(8.5)$ & 0.549 \\
\hline Total & 28 & $(100.0)$ & 31 & $(100.0)$ & 59 & $(100.0)$ & \\
\hline \multicolumn{8}{|l|}{ Performs anthropometry } \\
\hline 0 & 3 & $(11.1)$ & 7 & $(22.6)$ & 10 & $(17.2)$ & \\
\hline 1 & 24 & $(88.9)$ & 24 & $(77.4)$ & 48 & $(82.8)$ & 0.212 \\
\hline Total & 27 & $(100.0)$ & 31 & $(100.0)$ & 58 & $(100.0)$ & \\
\hline \multicolumn{8}{|l|}{ Performs dietary anamnesis } \\
\hline 0 & 26 & $(92.9)$ & 29 & $(93.5)$ & 55 & $(93.1)$ & \\
\hline 1 & 2 & $(7.1)$ & 2 & $(6.5)$ & 4 & $(6.9)$ & 0.654 \\
\hline \multirow[b]{4}{*}{ Continuous variables } & 28 & $(100.0)$ & 31 & $(100.0)$ & 59 & $(100.0)$ & \\
\hline & \multicolumn{4}{|c|}{ Groups } & & & \\
\hline & \multicolumn{2}{|c|}{ Control } & \multicolumn{2}{|c|}{ Intervention } & & & \\
\hline & $\mathbf{n}$ & Median & $\mathbf{n}$ & Median & \multicolumn{2}{|c|}{ No answer } & $\mathbf{p}^{+}$ \\
\hline Age of professional (years) & 26 & 46 & 31 & 46 & \multicolumn{2}{|c|}{2} & 0.975 \\
\hline Time since qualified (years) & 28 & 22 & 31 & 23 & \multicolumn{2}{|c|}{-} & 0.988 \\
\hline $\begin{array}{l}\text { Experience in infant and young } \\
\text { child feeding (years) }\end{array}$ & 22 & 19 & 27 & 20 & \multicolumn{2}{|c|}{-} & 0.762 \\
\hline Performance* (from 0 to 82 ) & 28 & 39 & 30 & 37 & \multicolumn{2}{|c|}{1} & 0.651 \\
\hline Feeding* (from 0 to 22 ) & 28 & 09 & 31 & 09 & \multicolumn{2}{|c|}{-} & 0.865 \\
\hline Actions* (from 0 to 18 ) & 28 & 12 & 31 & 11 & \multicolumn{2}{|c|}{-} & 0.476 \\
\hline $\begin{array}{l}\text { Behavior of mother }{ }^{\neq} \text {(from } 0 \text { to } \\
\text { 15) }\end{array}$ & 28 & 07 & 31 & 08 & \multicolumn{2}{|c|}{-} & 0.667 \\
\hline
\end{tabular}

* Fisher's exact test.

+ Kruskal-Wallis test.

* Measured before intervention. 


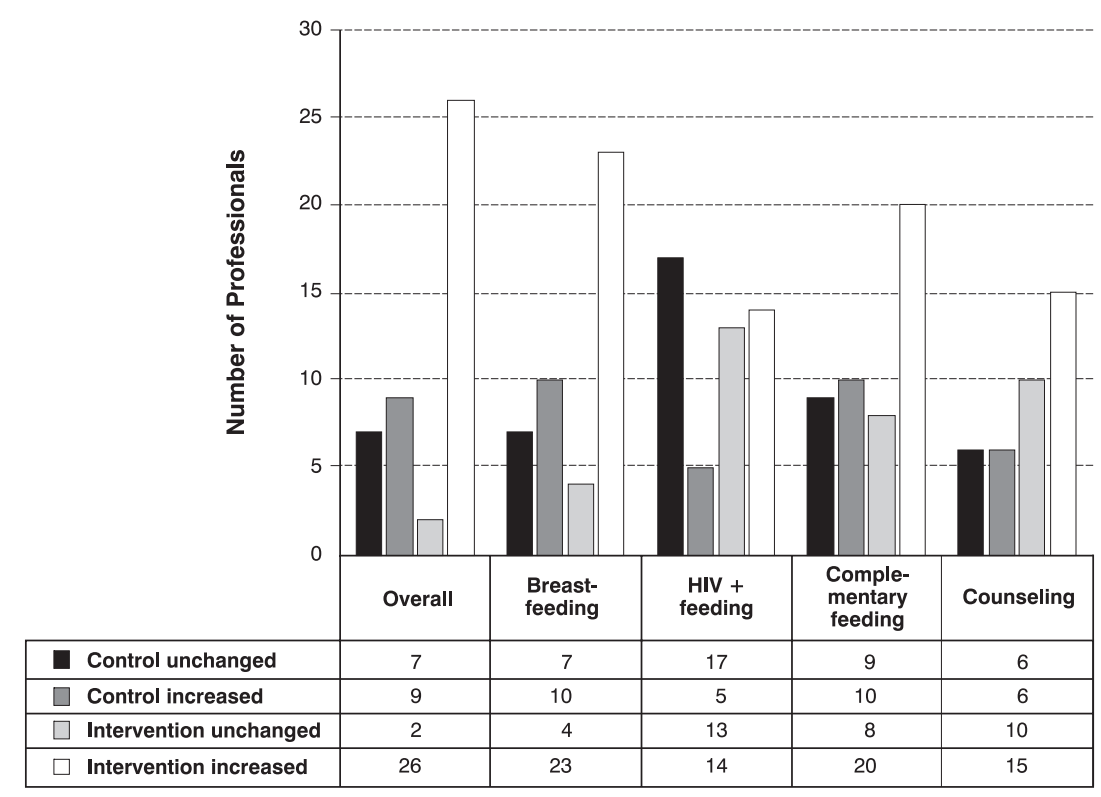

Figure 1 - Comparisons between the performance of the professionals in the control and intervention groups in terms of overall knowledge and information on breastfeeding, HIV and infant and young child feeding, complementary feeding and counseling (São Paulo, SP, Brazil, 2005)

With relation to domain A (counseling skills), the data suggest that variations were equal in the groups at each point, before (1) and after the intervention (2). The scores for this domain are mildly inferior at the second point in the IG. For domain B (dietary anamnesis), in general, when the groups were compared at each point, apparently, before training the professionals exhibited the same performance, on average. However, after training, the mean scores of those professionals who had been trained indicate improved performance (Figure 1).

With relation to domain $C$ (nutritional assessment and guidance), in general it was observed that, on average, the CG exhibited evaluation and guidance scores that were worse than the IG at both observation points.

With relation to the results of domain $D$ (reaction of mother to professional), it was observed that the mean scores are very close together and that the CG exhibits a mild increase in scores for mothers' reaction to the professional between points 1 and $2 *$; in the IG the opposite took place: the scores at point $2^{*}$ demonstrate a tendency for the scores of those who went on the Integrated Course to improve. However, considering the variability observed, these figures cannot really be said to be different.

Analysis of variance for repeated measures was used to compare the behavior of the groups during clinical consultations. ${ }^{22}$ Domain B was the only area to exhibit a statistically significant interaction between observation points and groups $(p<0.001)$. Therefore, it can be stated that the groups behaved differently over time in relation to dietary anamnesis (Figure 2).

The results of multiple comparisons allow us to state that the only statistically significant differences detected occurred when comparing points 1 and $2^{*}$ for the IG $(p=0.032)$ and when comparing CG and IG at point $2 *(p=0.008)$. In other words, while the mean CG scores were constant over time, the IG scores improved (Table 2).

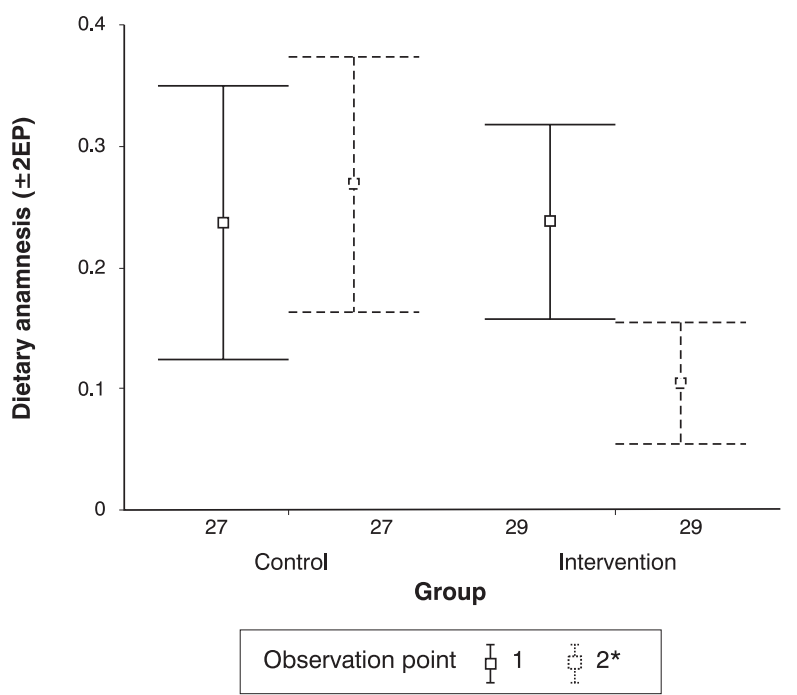

Figure 2 - Mean professional performance profiles during consultations, for dietary anamnesis, by group (intervention or control) (São Paulo, SP, Brazil, 2005) 
Table 2 - Summary means of the performance of the professionals during consultations descriptive levels of the analysis of variance employed for each of the domains observed, by group (intervention or control) (São Paulo, SP, Brazil, 2005)

\begin{tabular}{|c|c|c|c|c|c|c|c|c|}
\hline \multirow{2}{*}{\multicolumn{2}{|c|}{ Domain }} & \multirow[b]{2}{*}{ Time } & \multirow[b]{2}{*}{ Group } & \multirow[b]{2}{*}{ Mean } & \multirow[b]{2}{*}{ Standard deviation } & \multicolumn{3}{|c|}{ Source of variation } \\
\hline & & & & & & Time & Group & $\begin{array}{l}\text { Time- } \\
\text { Group }\end{array}$ \\
\hline \multirow[t]{4}{*}{ A) } & $\begin{array}{l}\text { Counseling } \\
\text { skills }\end{array}$ & 1 & Control & 2.42 & $0.59(1.53-3.88)$ & & & \\
\hline & & & Intervention & 2.38 & $0.51(1.59-4.12)$ & & & \\
\hline & & $2 *$ & Control & 2.22 & $0.52(1.38-3.71)$ & $<0.001$ & 0.225 & 0.122 \\
\hline & & & Intervention & 1.96 & $0.50(1.27-2.85)$ & & & \\
\hline \multirow[t]{4}{*}{ B) } & Dietary & 1 & Control & 0.24 & $0.29(0.00-0.86)$ & & & \\
\hline & & & Intervention & 0.24 & $0.22(0.00-0.71)$ & & & \\
\hline & & $2 *$ & Control & 0.27 & $0.27(0.00-0.83)$ & 0.137 & 0.131 & 0.018 \\
\hline & & & Intervention & 0.10 & $0.13(0.00-0.50)$ & & & \\
\hline \multirow[t]{4}{*}{ C) } & $\begin{array}{l}\text { Nutritional assessment } \\
\text { and guidance }\end{array}$ & 1 & Control & 0.53 & $0.22(0.00-0.89)$ & & & \\
\hline & & & Intervention & 0.44 & $0.23(0.00-0.86)$ & & & \\
\hline & & $2 *$ & Control & 0.52 & $0.14(0.27-0.78)$ & 0.906 & 0.042 & 0.996 \\
\hline & & & Intervention & 0.44 & $0.19(0.13-1.00)$ & & & \\
\hline \multirow[t]{4}{*}{ D) } & Mothers' reactions & 1 & Control & 2.44 & $0.69(1.00-4.00)$ & & & \\
\hline & & & Intervention & 2.44 & $0.75(1.00-3.67)$ & & & \\
\hline & & $2 *$ & Control & 2.48 & $0.60(1.17-3.83)$ & 0.830 & 0.674 & 0.605 \\
\hline & & & Intervention & 2.36 & $0.57(1.50-3.83)$ & & & \\
\hline
\end{tabular}

* Observation point 2: mean of the two observations carried out after the intervention.

Control: $\mathrm{n}=27$; Intervention: $\mathrm{n}=29$.

\section{Discussion}

The results of this study demonstrate that the Integrated Course was effective in increasing the knowledge of professionals about counseling in infants and young child feeding, breastfeeding and complementary feeding.

In terms of changes in professional performance resulting from the intervention, the results demonstrate significant improvement only in the practice of dietary anamnesis, and not in counseling, mothers reactions or nutritional assessment and guidance. In the case of counseling, which was the primary object of this intervention, it is worth noting that there was some improvement in the IG, although not a significant one.

It is important, however, to take into account certain limitations of this study: the sample that was initially expected suffered losses, which took place at different stages, and which are understandable in the context, not entirely favorable, of the city of São Paulo and its health system. Although we did restrict ourselves to the number of participants recommended by the course itself and even though randomization was successful, it was not possible to maintain equal representation of the different professional categories (pediatricians and nutritionist). In the final configuration, this disproportion was of 23 nutritionists to 33 pediatricians greater in the CG than in the IG. Although it had not been our objective to compare the behavior of these categories, it cannot be ignored that there are significant differences, both in terms of training and in terms of daily practice relating to infant and young child feeding, and, having restricted the study to these categories, any discussion of how other professional categories would react to the same intervention is ruled out.

One could also question whether the losses were professionals who were less interested in the themes of the Integrated Course, which would have biased our results to more; 
but one could equally imagine the opposite, that the losses included many who already knew about the theme and were therefore uninterested in studying further, which would have biased our results to "less." Since the data on the professionals who dropped out or refused is not available, it is not possible to state what type of bias may have existed, if any.

This was the first opportunity at which the Integrated Course has been assessed, with its current format and purpose. There are, however, in the literature studies with analogous references, whose results could be compared. ${ }^{7,10-13,15,23-26}$

Another study carried out with professionals from São Paulo, aiming to assess the WHO/UNICEF Breastfeeding Counseling Course demonstrated a significant improvement in counseling skills. In contrast with our study, however, that one was developed to deal solely with the theme of breastfeeding. It was observed that, immediately after the course, the participants' knowledge and skills in clinical management of breastfeeding and counseling significantly improved, in relation to those of the control group, and that this was repeated 3 months after intervention. ${ }^{15}$

Similar findings can be found in the results of studies undertaken in the United Kingdom, ${ }^{12}$ Bangladesh, ${ }^{10} \mathrm{Ghana}^{7}$ Mexico ${ }^{13}$ and Brazil, ${ }^{11}$ but always in interventions that, while including counseling, give priority to just one feature (or phase of life) of child nutrition, without including special dietary situations, such as HIV/AIDS. With relation to the Integrated Course, the subject of this study, while it may be considered that there were few gains in counseling skills, the good results achieved in general knowledge on infant and young child feeding and dietary anamnesis may represent a welcome improvement to what is currently found in daily practice.

This evidence emphasizes the importance of carrying out actions relating to counseling on infant and young child feeding that integrate content, in the form of permanent practices, to child healthcare policy, highlighting concern with constantly updating professional's training.

The fact that this project was carried out with health professionals who are already working for a large scale institution such as the DH/SP and in a city with the Metropolitan characteristics of São Paulo, demonstrates its potential for implementation in and adaptation to more circumspect circumstances.

\section{Acknowledgements}

We are grateful to Tereza Setsuko Toma, for coordinating the Integrated Course; to Ausônia Favorido Donato, for help with the theoretical background in education and with defining the methodology used to assess the facilitators; to Denise Pimentel Bergamaschi, for help with part of the statistical analysis and to José Martines (WHO), for help with the methodological design used in the study.

\section{References}

1. Patterson LE, Eisenberg S. O processo de aconselhamento. São Paulo: Martins Fontes; 2003.

2. Brasil, Ministério da Saúde. Aconselhamento em DST, HIV e AIDS. $2^{a}$ ed. Brasília: Ministério da Saúde; 1998.

3. World Health Organization.Breastfeeding counselling: a training course. Geneva: WHO; 1994.

4. World Health Organization. HIV and infant feeding counselling: a training course. Geneva: WHO; 2000.

5. World Health Organization.Complementary feeding counselling: a training course. Geneva: WHO; 2004.

6. World Health Organization. Infant and young child feeding counselling: an integrated course. Geneva: WHO; 2006.

7. Aidam BA, Perez-Escamilla R, Lartey A. Lactation counseling increases exclusive breast-feeding rates in Ghana. J Nutr. 2005; 135:1691-5.

8. Arranz P, Ulla SM, Ramos JL, Del Rincon C, Lopez-Fando T. Evaluation of a counseling training program for nursing staff. Patient Educ Couns. 2005;56:233-9.

9. Coutinho SB, Lima MC, Ashworth A, Lira PI. Impacto de treinamento baseado na Iniciativa Hospital Amigo da Criança sobre práticas relacionadas a amamentação no interior do Nordeste. J Pediatr (Rio J). 2005;81:471-7.

10. Haider R, Kabir I, Hamadani JD, Habte D. Reasons for failure of breast-feeding counselling: mothers' perspectives in Bangladesh. Bull World Health Organ. 1997;75:191-6.

11. Leite AJ, Puccini RF, Atalah AN, Alves Da Cunha AL, Machado MT. Effectiveness of home-based peer counselling to promote breastfeeding in the northeast of Brazil: a randomized clinical trial. Acta Paediatr. 2005;94:741-6.

12. Moran VH, Bramwell R, Dykes F, Dinwoodie K. An evaluation of skills acquisition on the WHO/UNICEF Breastfeeding Management Course using the pre-validated Breastfeeding Support Skills Tool (BeSST). Midwifery. 2000;16:197-203.

13. Morrow AL, Guerrero ML, Shults J, Calva JJ, Lutter C, Bravo J, et al. Efficacy of home-based peer counselling to promote exclusive breastfeeding: a randomised controlled trial. Lancet. 1999;353:1226-31.

14. Pelto GH, Santos I, Gonçalves H, Victora C, Martines J, Habicht JP. Nutrition counseling training changes physician behavior and improves caregiver knowledge acquisition. J Nutr. 2004; 134:357-62.

15. Rea MF, Venancio SI. Avaliação do curso de aconselhamento em amamentação OMS/UNICEF. J Pediatria (Rio J). 1999;75:112-8.

16. Santos IS, Victora CG, Martines J, Gonçalves H, Gigante DP, Valle $\mathrm{NJ}$, et al. Avaliação da eficácia do aconselhamento nutricional dentro da estratégia do AIDPI (OMS/UNICEF). Rev Bras Epidemiol. 2002; 5:15-29.

17. Stefanelli MC. Comunicação com paciente: teoria e ensino. $2^{a}$ ed. São Paulo: Robe; 1993.

18. Claeson M, Gillespie D, Mshinda H, Troedsson H, Victora CG; Bellagio Study Group on Child Survival. Knowledge into action for child survival. Lancet. 2003;362:323-7.

19. Garrick C. Teaching for counselling skills. Med J Aust. 1979; 2:358-9.

20. Kerr FRM. A relação médico-paciente e a qualidade do atendimento médico [dissertação]. São Paulo: Faculdade de Saúde Pública da Universidade de São Paulo; 2000. 
82

21. Leite AM, Silva IA, Scochi CG. Comunicação não-verbal: uma contribuição para o aconselhamento em amamentação. Rev Latino-Am Enfermagem. 2004;12:258-64.

22. Winer B. Statistical principles in experimental design. New York: McGraw-Hill; 1971.

23. Albernaz E, Giugliani ER, Victora CG. Supporting breastfeeding: a successful experience. J Hum Lact. 1998;14:283-5.

24. Chopra M, Doherty T, Jackson D, Ashworth A. Preventing HIV transmission to children: quality of counselling of mothers in South Africa. Acta Paediatr. 2005;94:357-63.

25. Santiago LB, Bettiol H, Barbieri MA, Guttierrez MR, Del Ciampo LA. Incentivo ao aleitamento materno: a importância do pediatra com treinamento especifico. J Pediatr (Rio J). 2003; 79:504-12.
Infant and young child feeding counseling - Bassichetto KC \& Réa MF

26. Valle NJ, Santos I, Gigante DP, Gonçalves H, Martines J, Pelto $\mathrm{GH}$. Household trials with very small samples predict responses to nutrition counseling intervention. Food Nutr Bull. 2003; 24:343-9.

Correspondence:

Katia Cristina Bassichetto

Rua Gal. Jardim, 36, $5^{\circ}$ andar, Vila Buarque

CEP 01223-010 - São Paulo, SP - Brazil

Tel.: +55 (11) 3218-4084

E-mail: kcbassi@gmail.com 